\title{
Treatment of Wastewater Dyeing Agent by Photocatalytic Process in Solar Reactor
}

\author{
O. Zahraa, ${ }^{1}$ S. Maire, ${ }^{1}$ F. Evenou, ${ }^{1}$ C. Hachem, ${ }^{1,2}$ M. N. Pons, ${ }^{3}$ A. Alinsafi, ${ }^{1,4}$ and M. Bouchy ${ }^{1}$ \\ ${ }^{1}$ Département de Chimie Physique des Réactions, UMR 7630 CNRS-INPL, ENSIC, 1 rue Grandville, BP 20451, \\ 54001, Nancy Cedex, France \\ ${ }^{2}$ Faculty of sciences, Damascus University, P.O. BOX 5665, Damascus, Syria \\ ${ }^{3}$ Laboratoire des Sciences du Génie Chimique, UPR 6811 CNRS-INPL, ENSIC, 1 rue Grandville, BP 20451, \\ 54001, Nancy Cedex, France \\ ${ }^{4}$ Laboratoire d'Automatique et d'Etude des Procédés, Faculté des Sciences Semlalia, Université Cadi Ayyad, \\ Avenue Prince Moulay Abdellah, BP 2390, 40000 Marrakech, Morocco
}

Received 31 March 2006; Accepted 7 April 2006

\begin{abstract}
The photocatalytic decolorization of industrial textile dyes has been studied. The treatment was carried out on a solar reactor consisting in a flat active plane, tilted so as to face the sun and to allow the trickling of the water to be treated. Alternatively the reactor could be irradiated by an artificial source. After checking the system using salicylic acid, a conventional model molecule, the photocatalytic decolorization of Orange II, Yellow Drimarene, and Black Drimarene dyes was investigated. Artificial and solar irradiation gave comparable results although the heating by the sun reduced the amount of adsorption. The kinetics agrees with the Langmuir-Hinshelwood model and a discrepancy between adsorption constants deduced from the kinetic and adsorption experiments was interpreted by considering various types of adsorption sites. Orange II and Drimarene dyes decolorization kinetics are opposite limiting cases of the above model, as being of order 0 and 1 with respect to the dye, respectively.
\end{abstract}

Copyright (c) 2006 O. Zahraa et al. This is an open access article distributed under the Creative Commons Attribution License, which permits unrestricted use, distribution, and reproduction in any medium, provided the original work is properly cited.

\section{INTRODUCTION}

As a result of industrial activities and domestic utilities, water consumption has been widely increased in the last decade. Consequently, the reserve of water, particularly in North Africa, has considerably decreased. Moreover, in many of these countries, textile industry is very developed. Therefore, water consumption and pollution could be indirectly enhanced, which necessitate the presence of a wastewater treatment. During the last two decades, research on water treatment has progressed continuously in an exponential manner, in order to satisfy the rigorous requirement on the quality of water. Thus, advanced oxidation processes (AOP) have been extensively investigated, regarding the degradation of pollutants [1-3]. In particular, heterogeneous photocatalysis appears today as an interesting application of $\mathrm{AOP}$, leading to a complete mineralization of most organic compounds. In this process, hydroxyl radicals $\mathrm{HO}^{-}$are generated when the photocatalyst is illuminated in presence of water and air [4-6]. These ultra reactive species associated with oxygen are able to achieve a complete mineralization of organic pollutants into carbon dioxide, water, and other nontoxic products $[7,8]$.
In this work, the photocatalytic decolorization and degradation of several dyes has been investigated, under both artificial and solar irradiations so as to assess the efficiency of the process and the effect of various physico-chemical parameters.

\section{EXPERIMENTAL PROCEDURES}

\subsection{Materials and methods}

Salicylic acid and the dye Orange II were commercial products purchased from Sigma Chemical Co., and used without further purification. Drimarene Yellow DR-K2R and Drimarene Black DR-S-Np150 were provided by CLARIANT (La Defense, France). Purified water was obtained from Elix Millipore equipment. Titanium dioxide was Degussa "TITANDIOXID P25." According to the manufacturer's specifications [9], it was about $70 \%$ anatase and the elementary particle in dry powder was approximately spherical in shape with a size of $20 \mathrm{~nm}$. The specific surface area, as measured by $\mathrm{N}_{2}$ adsorption at $77 \mathrm{~K}$, was $44 \mathrm{~m}^{2} \mathrm{~g}^{-1}[10]$. 
UV fluorescent lamp (365 nm, $18 \mathrm{~W})$

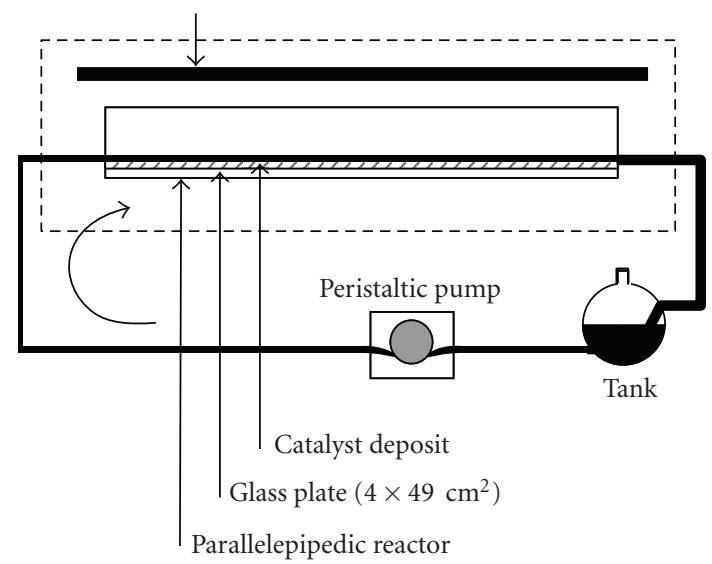

FIgURE 1: Laboratory experimental setup for the photocatalytic degradation.

Photocatalytic experiments were carried out on two types of reactors: a laboratory reactor using artificial light and a solar reactor using sun or artificial light. In both reactors, the aqueous solution was allowed to circulate, by use of a peristaltic pump, in a loop through the reactor and a tank where it was aerated. The solution flowrate was of $0.4 \mathrm{~L} \cdot \mathrm{min}^{-1}$ for a total volume of solution of $200 \mathrm{~cm}^{3}$ which insured a residence time of $0.5 \mathrm{~min}$ per pass. The principle experimental setup is shown in Figure 1 for the laboratory reactor [11].

The laboratory setup consisted in a horizontal parallelepiped tank of dimensions $3.8 \times 48 \mathrm{~cm}^{2}$. The catalyst was supported on glass plate fitting the bottom of the reactor according to the procedure given below. Irradiation was obtained by an 18 W UV "black light" fluorescent lamp MAZDA-TFN-18.

The solar setup made use of a flat-plate reactor (Figure 2) of dimensions $30 \times 30 \mathrm{~cm}^{2}$, tilted at an angle of 30 degrees with respect to the horizontal plane, where the solution was allowed to trickle down freely from a pipe pierced by several openings placed at the top of the reactor. The base of the reactor was made of aluminium and covered with a glass plate. The catalyst was either a set of plates as described above (see Figure 2) or a photocatalytic material graciously supplied by AHLSTROM and which is described below. When exposed to solar irradiation, the reactor was placed southwards and worked in June around noon. Under these conditions, the reactor was nearly facing the sun. Alternatively, artificial irradiation was obtained by two fluorescent lamps as described above, placed parallel to the plate as shown in Figure 2. After each run, the catalyst was washed and irradiated for several hours in the presence of $\mathrm{H}_{2} \mathrm{O}_{2}$ to prevent fouling.

Salicylic acid concentration was monitored by HPLC on a chromatograph equipped with a Shimadzu SPD-6A spectrophotometric detector. A Lichrosob column was used and elution was carried out by a mixture of methanol and water. In dye decolorization experiments, absorption measurements were carried out on a Lambda-2 PERKIN-ELMER UV/VIS spectrophotometer. The analysis wavelengths, corresponding to the absorption maximum were 485, 390,

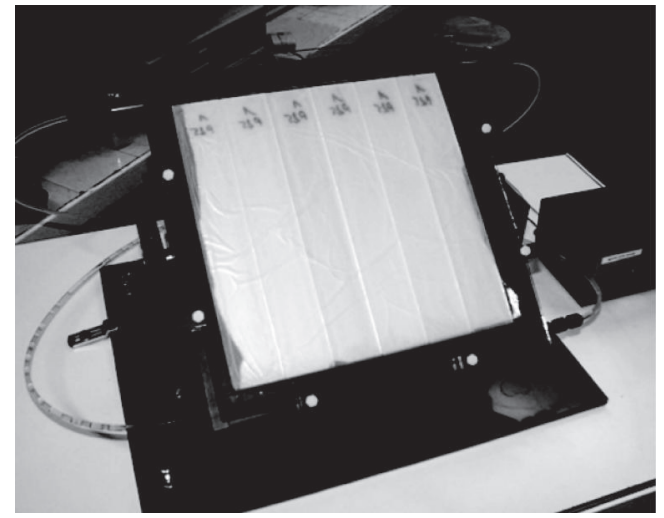

(a)

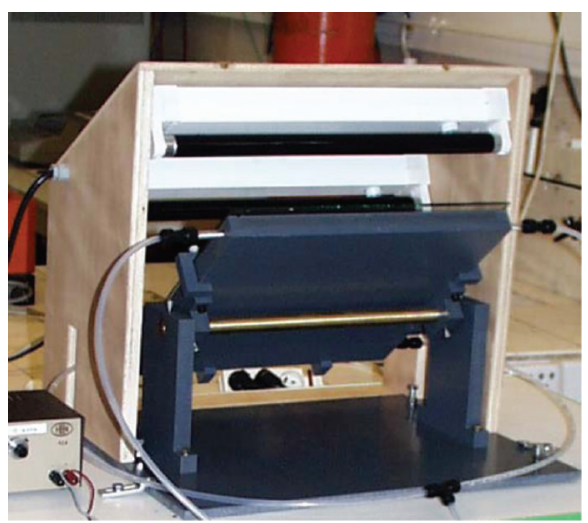

(b)

FIGURE 2: Solar photocatalytic reactor and setup for artificial irradiation.

and $600 \mathrm{~nm}$ for the monitoring of Orange II, Drimarene Yellow, and Drimarene Black, respectively, with a measured extinction coefficient of $2.2610^{4} \mathrm{~L} \cdot \mathrm{mol}^{-1} \mathrm{~cm}^{-1}, 2.33$ $10^{-2} \mathrm{~L} \cdot \mathrm{mg}^{-1} \mathrm{~cm}^{-1}$, and $1.8510^{-2} \mathrm{~L} \cdot \mathrm{mg}^{-1} \mathrm{~cm}^{-1}$, respectively.

Electron microscopy of the deposited catalyst was carried out on a JEOL T33ФA scanning microscope.

\subsection{Catalyst}

In order to carry out the deposition of catalyst on a glass plate, a suspension of commercial Degussa P25 $\mathrm{TiO}_{2}$ of $4 \mathrm{~g} . \mathrm{L}^{-1}$ in pure water was prepared. $\mathrm{pH}$ was adjusted to about 3 and it was sonicated. A given volume suspension was carefully poured on the glass plate and allowed to dry out at $100^{\circ} \mathrm{C}$ for a few hours. After drying, the plate was fired at $475^{\circ} \mathrm{C}$ for 4 hours. During the heating, $\mathrm{OH}$ groups from the catalyst surface and the support can react and lose a molecule of water, creating an oxygen bridge, thus increasing the adherence of the catalyst to the support. Before deposition, the glass surface was treated using a dilute acid solution of HF and washed in a basic solution of $\mathrm{NaOH}$ in order to increase the number of $\mathrm{OH}$ groups. The suspension concentration $\left(4\right.$ g. $\left.\mathrm{L}^{-1}\right)$ was chosen so as to get thin enough deposits. This 


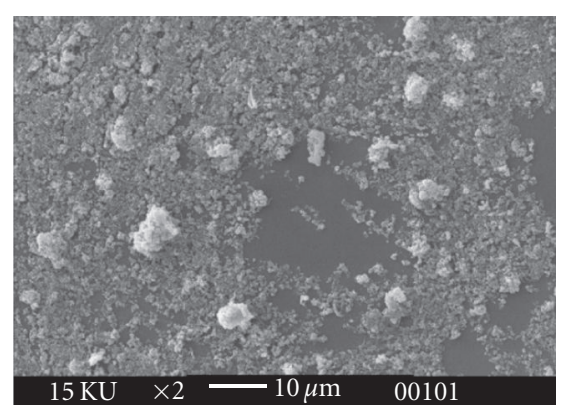

(a)

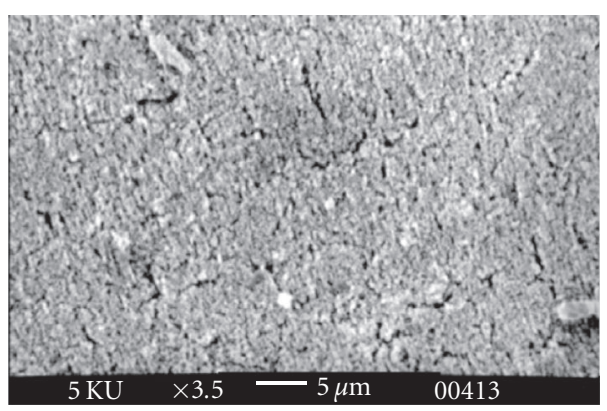

(b)

FIgURE 3: SEM of $\mathrm{TiO}_{2}$ P25 deposited on a glass plate.

deposition process could be carried several times in succession so as to increase the total thickness. As shown in Figure 3, the first coat does not cover the entire surface but additional coats lead to a complete coverage.

SEM pictures of AHLSTROM catalyst support (cf. Figure 4(a)) show that the support consists in nonwoven glass fibres of diameter about $10 \mu \mathrm{m}$ randomly distributed and held together by a mineral binder. As shown in Figures $4(\mathrm{~b})$ and $4(\mathrm{c})$, the catalyst $\mathrm{TiO}_{2}$ is deposited on the support as crystals of the submicrometric size. The catalyst is scattered on the threads and most of it is fixed on the binder as a layer consisting of the aggregation of small crystals. Mass transfer process can reduce the rate of reaction for deposits on a flat surface but an efficient mass transfer is expected from this 3-dimensional support.

\section{SALICYLIC ACID PHOTOCATALYTIC DEGRADATION}

The photocatalytic degradation of salicylic acid (SA) was first studied as this compound has been much studied and can be considered as a standard. In these experiments, the solar reactor was used under artificial irradiation. SA was allowed to adsorb on the catalyst in the dark for about 40 minutes before starting irradiation at time 0 . The "initial" concentration $\mathrm{CO}$ is therefore slightly lower to the original concentration of the solution due to a partial adsorption (of about 10\%).

Figure 5 shows the decrease in relative concentration $\mathrm{C} / \mathrm{CO}$ with time during irradiation using the UV lamps.
There is some variation in the rate of degradation, but not a decrease due to a fouling of the catalyst. Experiments using solar irradiation are shown in Figure 6. The efficiency using UV lamp or solar irradiation under sunny conditions are comparable with about $90 \%$ of degradation in about 40 minutes. This shows that the artificial irradiation is a satisfactory simulation of solar irradiation. When using the lamps, the light flux could be estimated from the yield in UV emission given by the supplier (about 1/6) and the computation of the effective solid angle, giving a value of $9 \mathrm{~W} \cdot \mathrm{m}^{-2}$. In solar irradiation, assuming a sun flux of about $500 \mathrm{~W} \cdot \mathrm{m}^{-2}$ at ground level and a proportion of about $3 \%$ of effective UV emission, the flux on the reactor was estimated to be of the order of $15 \mathrm{~W} . \mathrm{m}^{-2}$. Solar irradiation is therefore higher than the artificial irradiation and consequently the degradation rate is also slightly higher as can be expected from this semiquantitative estimation of the solar flux.

Irradiation under various weather conditions is also shown in Figure 6. As expected, when the weather is overcast the degradation is much slower despite the fact that this type of reactor collects diffusive light as well as direct sunlight, compared to reactors equipped with a collecting optics such as a parabolic trough. On the other hand a slightly cloudy sky does not affect the reaction in a noticeable way. Therefore, a reliable working of the system can be expected in countries benefiting of a regular sunny weather despite of passing clouds.

\section{ORANGE II PHOTOCATALYTIC DECOLORIZATION}

Orange II, a dye commonly used in textile industry, is a diazo compound with a sulphonate group with the formula in Scheme 1.

Orange II decolorization has been studied under solar irradiation using the solar reactor fitted with the catalyst deposited on glass plates. The decolorization of the dye was followed by spectrophotometry. It could be observed that the disappearance of the dye led to a total decolorization, which shows that possible intermediates in the degradation do not absorb in the visible range. The experiment shown in Figure 7 shows the photocatalytic nature of the process as there is no measurable effect due to irradiation in the absence of catalyst.

The effect of dye concentration is shown in Figure 8. The change in relative concentration decreases when the initial concentration $C 0$ is increased. However, plotting the variation of the absolute initial rate against the initial concentration shows (cf. Figure 9) that the rate is nearly independent of the concentration, that is the kinetics is of zero order with respect to the dye. It is interesting to compare this effect of the concentration on both the rate of reaction and the adsorption. The dye concentration was followed after contact with the catalyst in the setup used for the kinetics measurements, without the irradiation, and the adsorbed quantity at equilibrium was deduced from the mass balance when a limiting value of the concentration was reached. Figure 10 shows that the adsorbed quantity is nearly proportional to the dye concentration. 


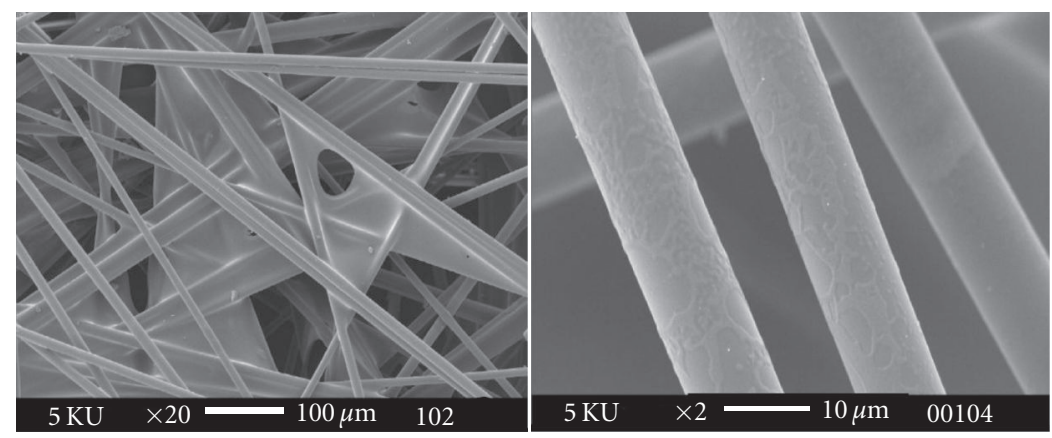

(a)
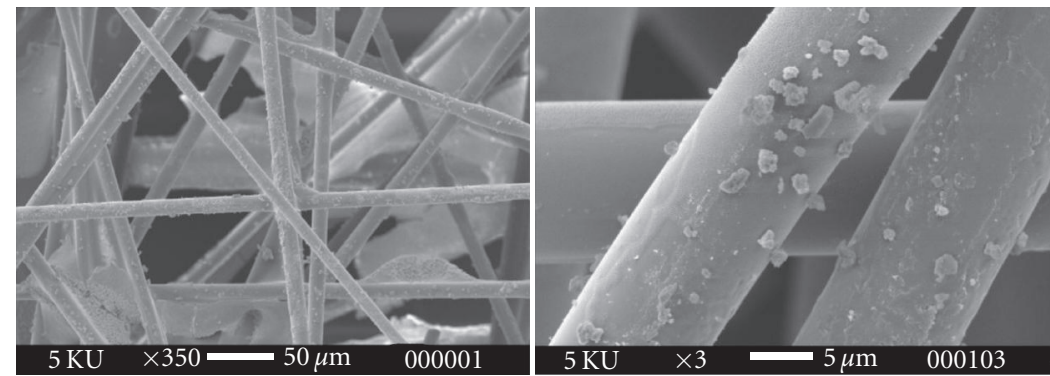

(b)

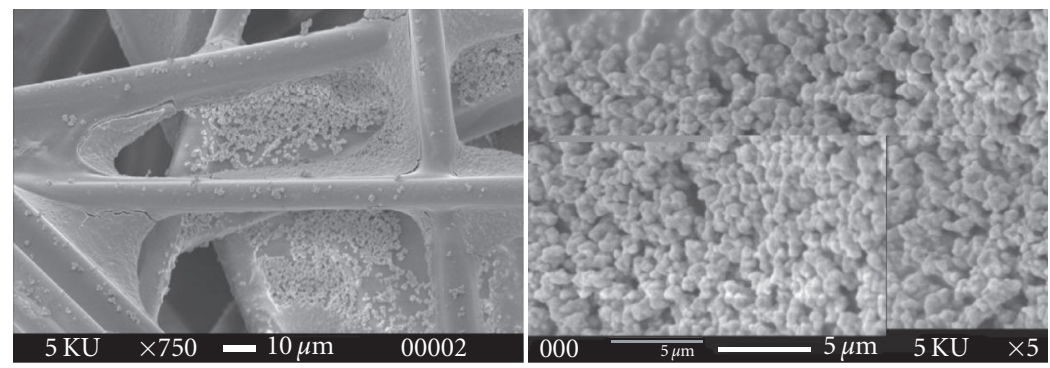

(c)

FIGURE 4: (a) SEM of Ahlstrom glass fibre support, (b) SEM of Ahlstrom impregnated support, deposition on fibres, and (c) SEM of Ahlstrom impregnated support, deposition on binder.

A common kinetic model is the Langmuir-Hinshelwood mechanism (LH) where the attack of the target molecule takes place on the catalyst surface where the molecule is adsorbed and reacts with either the light-activated catalyst itself (acting as electron donor or acceptor) or $\mathrm{HO}^{*}$ radicals formed on the surface from adsorbed water or hydroxide ions. The rate of reaction is assumed to be proportional to the adsorbed reactant, so that the kinetic law can be written for a given concentration $C$ :

$$
r=k \cdot\left[\frac{K_{\mathrm{LH}} \cdot C}{1+K_{\mathrm{LH}} \cdot C}\right],
$$

where $k$ is a rate constant, function of the irradiation flux, and $K_{\mathrm{LH}}$ the adsorption constant. This is derived from the Langmuir isotherm:

$$
Q=Q_{\max } \cdot\left[\frac{K_{L} \cdot C}{1+K_{L} \cdot C}\right]
$$

where $Q$ is the adsorbed quantity, $Q_{\max }$ the maximum value where the surface is saturated, and $K_{L}$ the adsorption constant. It has to be noticed that $K_{\mathrm{LH}}$ and $K_{L}$ are not identical (see below) and the value of $K_{\mathrm{LH}}$ is generally higher than the value of $K_{L}$ [12].

A zero order kinetics as observed here corresponds to a limiting case where the adsorption is practically maximal, that is $K_{\mathrm{LH}} \cdot C \gg 1$, leading to $r=k$.

A similar effect is therefore expected in the adsorbed quantity, that is $Q$ practically constant, equal to $Q_{\max }$, in the range of concentration considered, due to $K_{L} \cdot C \gg 1$ if the same adsorption takes place, that is $K_{\mathrm{LH}}=K_{L}$. Actually the opposite limiting case is observed, that is $Q$ nearly proportional to $C$, as shown in Figure 10, corresponding to $K_{L} \cdot C \ll 1$.

The apparent discrepancy between photocatalytic rate and adsorption can be due to the presence on the catalyst of various types of adsorption sites [13], in particular "active" 


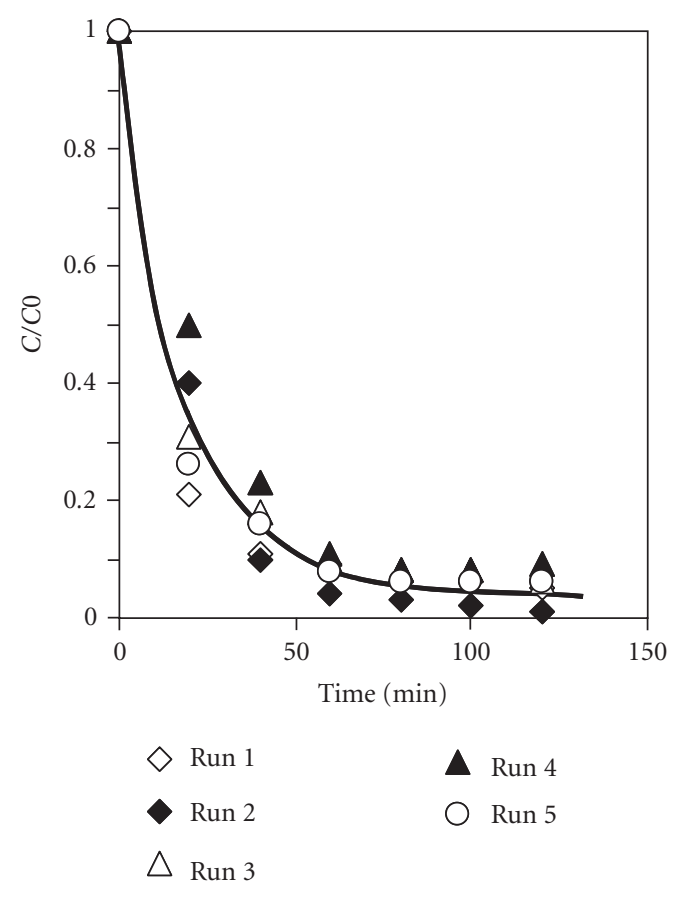

FIgURE 5: Salicylic acid degradation, UV lamp, $C 0=20 \mathrm{mgL}^{-1}$.

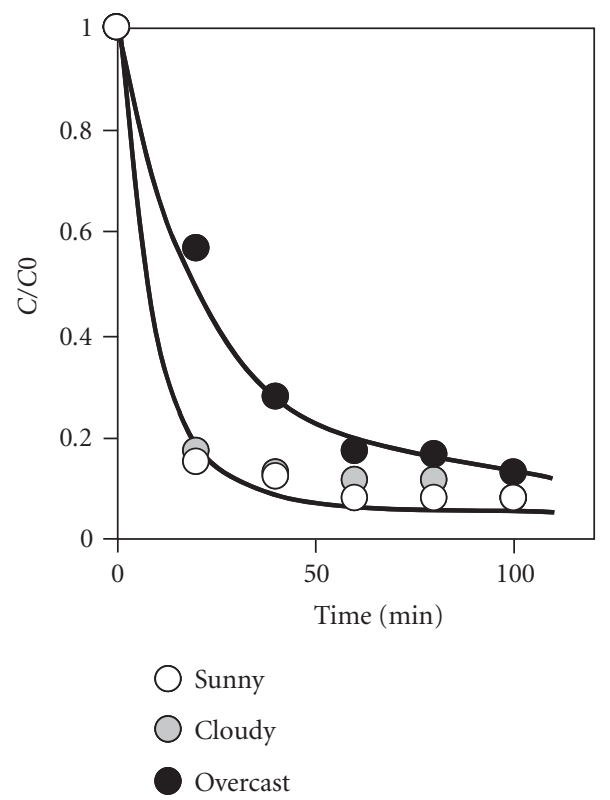

FIgURE 6: Salicylic acid degradation, solar irradiation, $C 0=$ $15 \mathrm{mgL}^{-1}$.

sites where the reaction can take place. On these active sites, the condition $K_{\mathrm{LH}} \cdot C \gg 1$ would be obeyed in the range of concentration considered due to a high adsorption constant $K_{\mathrm{LH}}$, whereas most sites possess a low adsorption constant $K_{L}$ leading to $K_{L} \cdot C \ll 1$. If the active sites are in small proportion, the observed adsorption is mainly due to the numerous nonreactive sites and the overall effect will verify

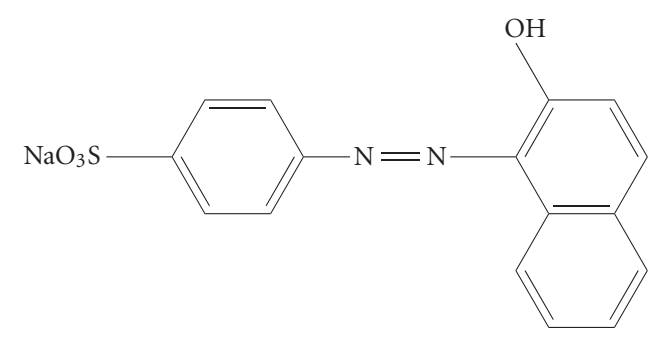

Scheme 1

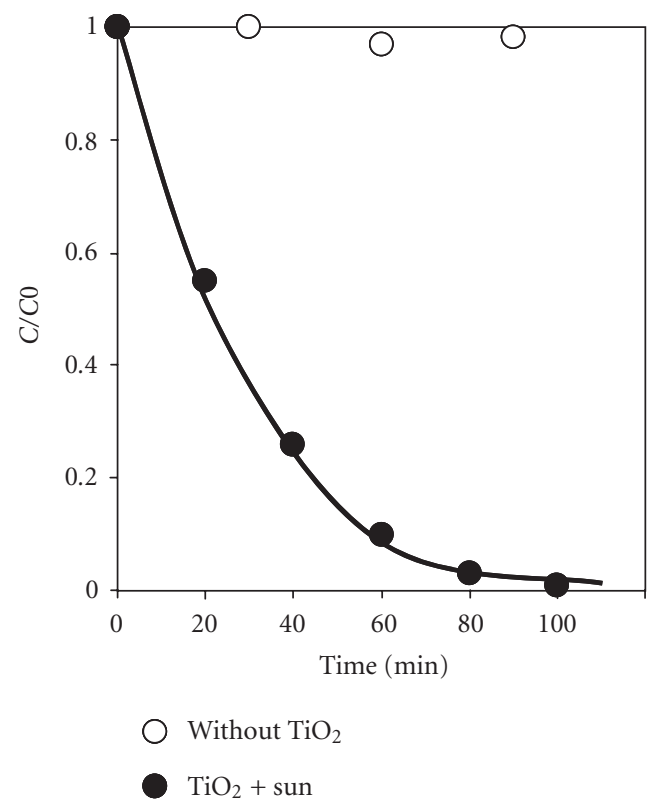

FIgURE 7: Solar photocatalytic decolorization of Orange II.

$K_{L} \cdot C \ll 1$. Such an effect explains an apparent discrepancy difference between the effect of concentration on the kinetics or the overall adsorption. Alternatively other phenomena have been also suggested such as photoadsorption to explain this discrepancy [14].

\section{DRIMARENE DYES PHOTOCATALYTIC DECOLORIZATION}

Drimarene dyes are currently used in textile industry. The dyes studied here are actual commercial samples, whose exact composition is not known. Basically, the decolorization of these dyes has been followed in a similar manner as the Orange II dye. However, the dyes have not been allowed to reach the adsorption equilibrium before irradiation and the irradiation was started immediately after filling the reactor. Therefore, the adsorption is very apparent on the graphs showing $\mathrm{C} / \mathrm{CO}$ as a function of time. On the first hand, the adsorption is much larger than in the preceding cases and on the second hand the adsorption is fast compared to the photocatalytic decolorization under the present conditions of irradiation. It therefore appears as a fast decrease in $C / C 0$ at initial times, 


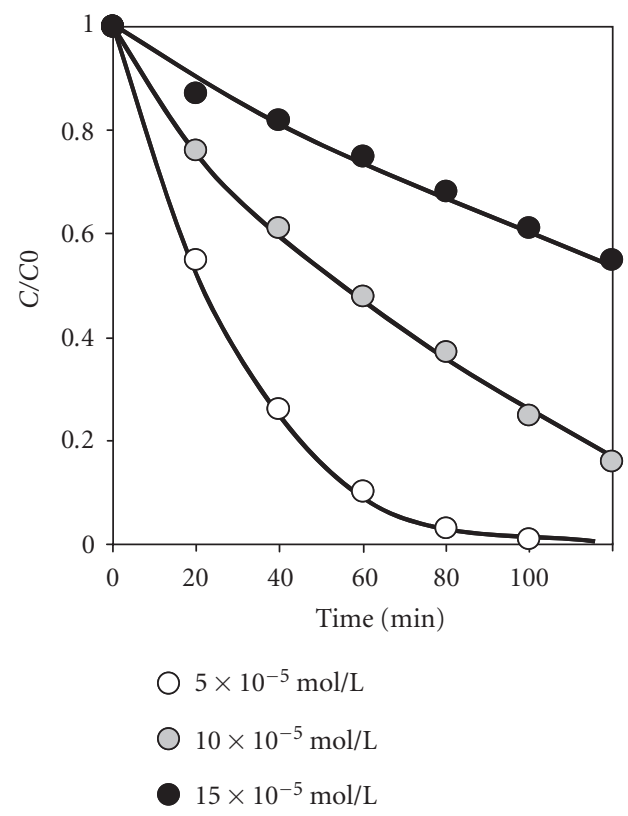

FIGURE 8: Solar photocatalytic decolorization of Orange II effect of the concentration.

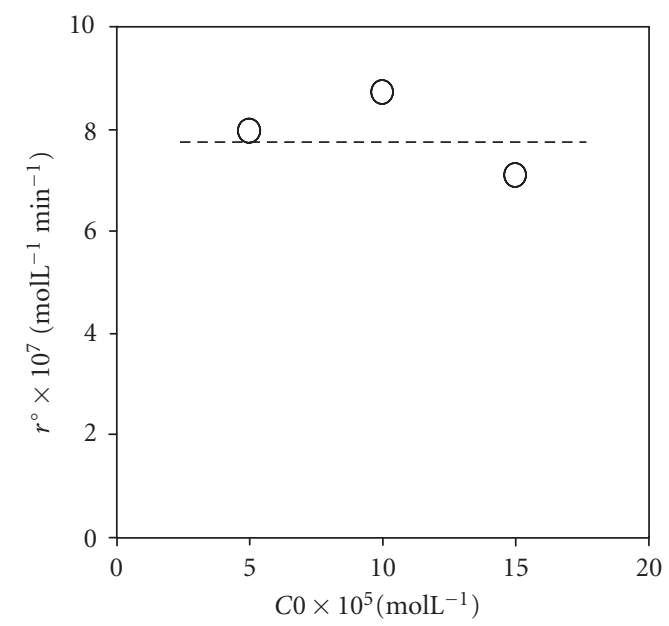

FIgURE 9: Solar photocatalytic decolorization of Orange II. Variation of the rate of disappearance with the concentration.

followed by a slower decrease due to the induced chemical degradation as shown for example in Figure 11.

Figure 11 shows the decolorization of Drimarene Yellow in the presence of catalyst under solar or artificial irradiation, as well as under artificial irradiation in the absence of catalyst. Contrary to Orange II, this dye exhibits a noticeable direct photodegradation in the absence of catalyst. It will be shown later that the Drimarene dyes do undergo efficient photocatalytic degradation in the presence of a higher mass of catalyst. The fast initial decrease in $\mathrm{C} / \mathrm{CO}$ at initial time is due to the adsorption. This adsorption is more important under artificial light than under solar irradiation. This is due to an artefact related to the temperature. Experiments under

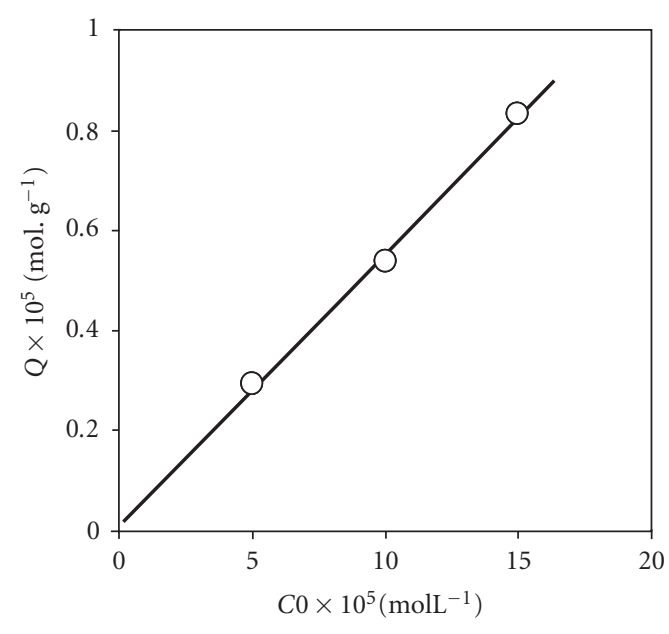

FIGURE 10: Photocatalytic decolorization of Orange II. Variation of the adsorption with the concentration.

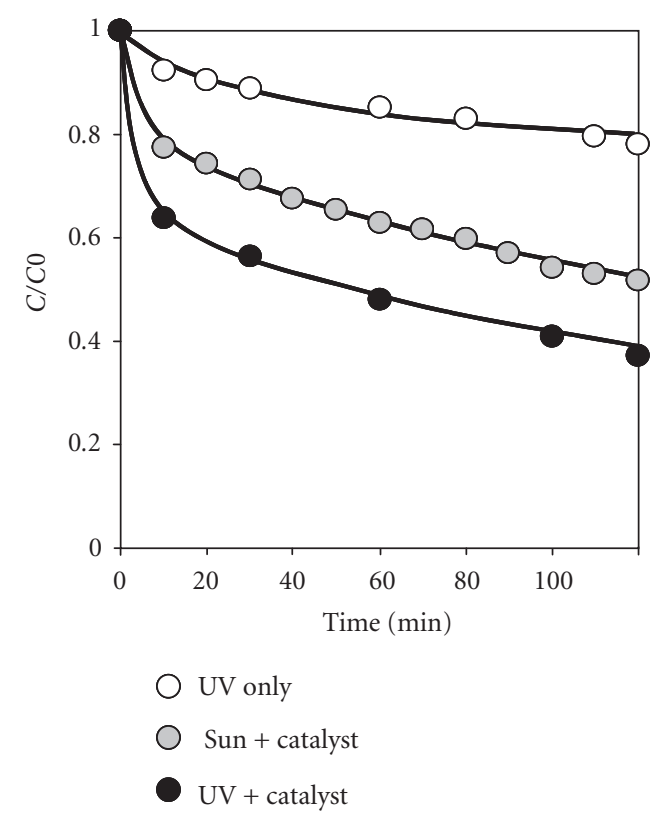

FIgURE 11: Photocatalytic decolorization of Drimarene Yellow, $\mathrm{CO}=25 \mathrm{mgL} \mathrm{L}^{-1}, \mathrm{TiO}_{2} 2.8 \mathrm{mg} \cdot \mathrm{cm}^{-2}$.

artificial light were conducted at room temperature; as the lamps are of low electric power and do not significantly heat the reactor. On the opposite, outdoors in July the temperature was around $35^{\circ} \mathrm{C}$, and solar irradiation, mainly in the infrared range, resulted in an important heating of the reactor, which reached a temperature of $40-45^{\circ} \mathrm{C}$ within few minutes after the beginning of the experiment, despite the circulation of the solution. Thus heating does not alter significantly the degradation; a common feature for light activated reactions, but has a strong effect on the adsorption, which decreases when the temperature is raised.

Decolorization of Drimarene Yellow and Drimarene Black are very similar as shown in Figure 12. It is slightly 


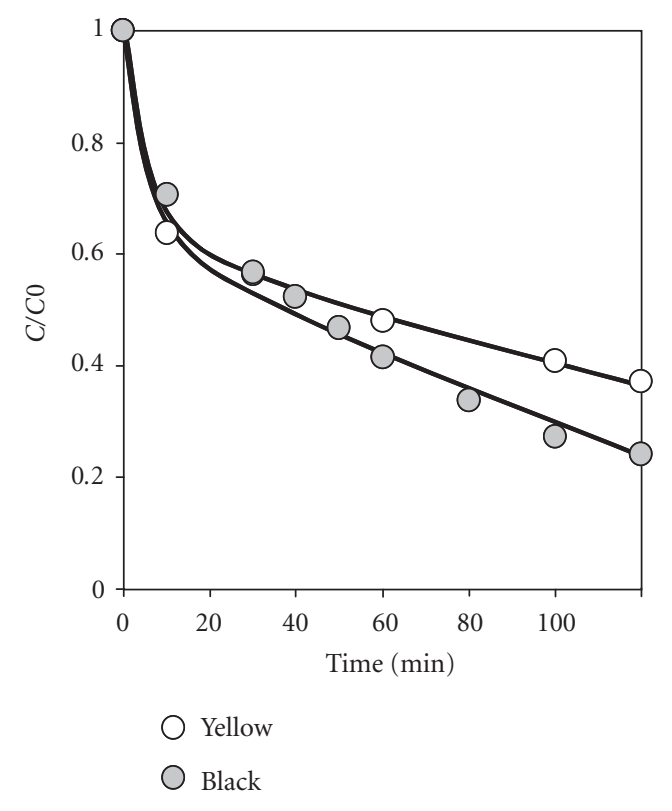

Figure 12: Photocatalytic decolorization of Drimarene Yellow and Drimarene Black (artificial irradiation), $\mathrm{C} 0=25 \mathrm{mgL}^{-1}, \mathrm{TiO}_{2}$ $2.8 \mathrm{mg} \cdot \mathrm{cm}^{-2}$.

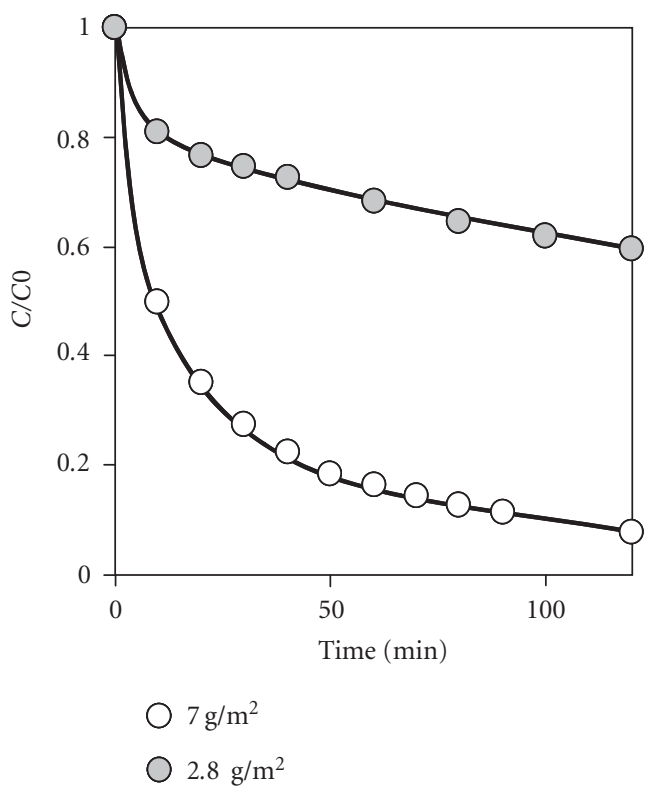

FIgURE 13: Photocatalytic decolorization of Drimarene Yellow (artificial irradiation), $C 0=25 \mathrm{mg} . \mathrm{L}^{-1}$, effect of catalyst mass.

more effective for Drimarene Black. Therefore, observations carried on one of the two dyes can be relevant for both.

A catalytic reaction can be improved by increasing the catalyst mass. As an example, the decolorization of Drimarene Yellow has been compared using two deposit thicknesses on the supporting glass plate. This is shown in Figure 13 where the rate of decolorization is roughly

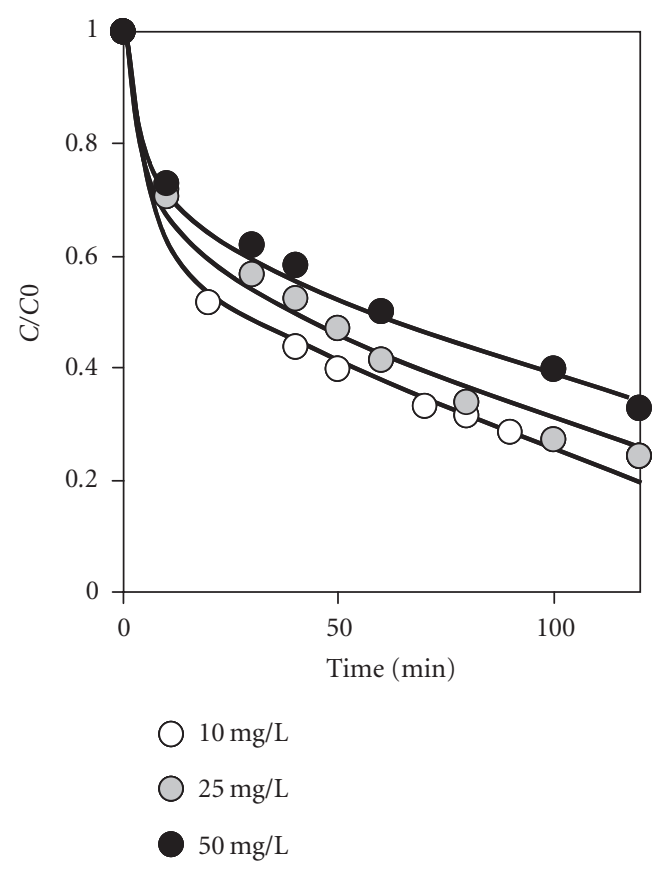

FIgURE 14: Photocatalytic decolorization of Drimarene Black (artificial irradiation), effect of the concentration, $\mathrm{TiO}_{2} 2.8 \mathrm{mg} \cdot \mathrm{cm}^{-2}$.

proportional to the catalyst mass. Note that such an increase cannot be expected on all range of mass, as in the case of conventional thermal catalysis, as the efficiency of the catalyst is related to the activation by absorbed light. For a given irradiation flux the rate is expected to reach a maximum value when all the light is absorbed as already shown elsewhere [12]. The dependence observed in the present case is due to a partial absorption of the light by the deposit in the range of thickness used in the experiment.

Figures 14 and 15 show the effect of the dyes concentration on the decolorization under artificial irradiation. Contrary to the case of Orange II, the decrease of $C / C O$ is practically independent on the concentration, which means that the absolute rate is nearly proportional to the dye concentration [15]. This first order with respect to the dye corresponds to a limiting case with $K_{\mathrm{LH}} \cdot C \ll 1$, that is the adsorption on the active sites is low, despite an important overall adsorption.

Under solar irradiation, the decolorization of Drimarene Yellow, shown in Figure 16, is similar to what is found under artificial irradiation. As observed previously in the case of Orange II, the adsorption is lower due to a rise in temperature. The rate of photocatalytic decolorization is lower under solar irradiation contrary to the case of Orange II. This can be partly due to a lower irradiation flux for the experiment has been carried out in September.

\section{CONCLUSION AND PERSPECTIVES}

Solar irradiation has proved an efficient source of light for the decolorization of dyes through a photocatalytic process and the transformation is practically complete in a reasonable 


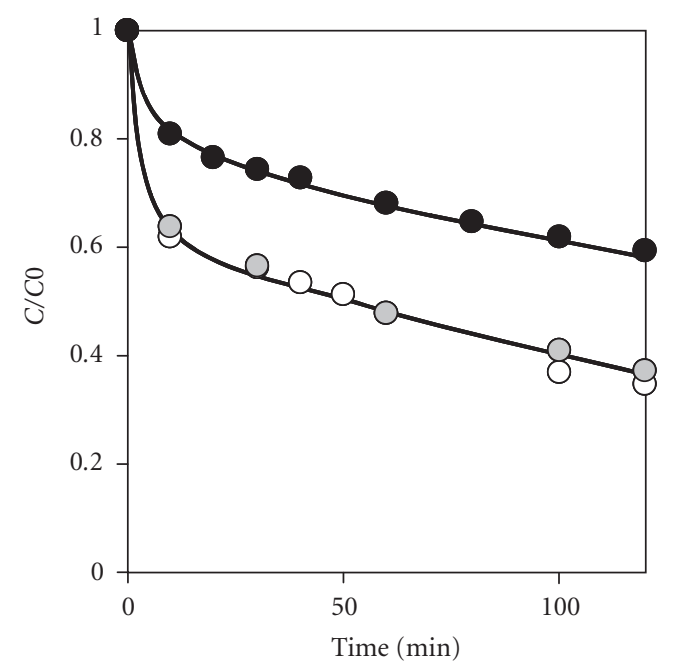

$$
\begin{aligned}
& 10 \mathrm{mg} / \mathrm{L} \\
& 25 \mathrm{mg} / \mathrm{L} \\
& 50 \mathrm{mg} / \mathrm{L}
\end{aligned}
$$

Figure 15: Photocatalytic decolorization of Drimarene Yellow (artificial irradiation), effect of the concentration, $\mathrm{TiO}_{2} 2.8 \mathrm{mg} \cdot \mathrm{cm}^{-2}$.

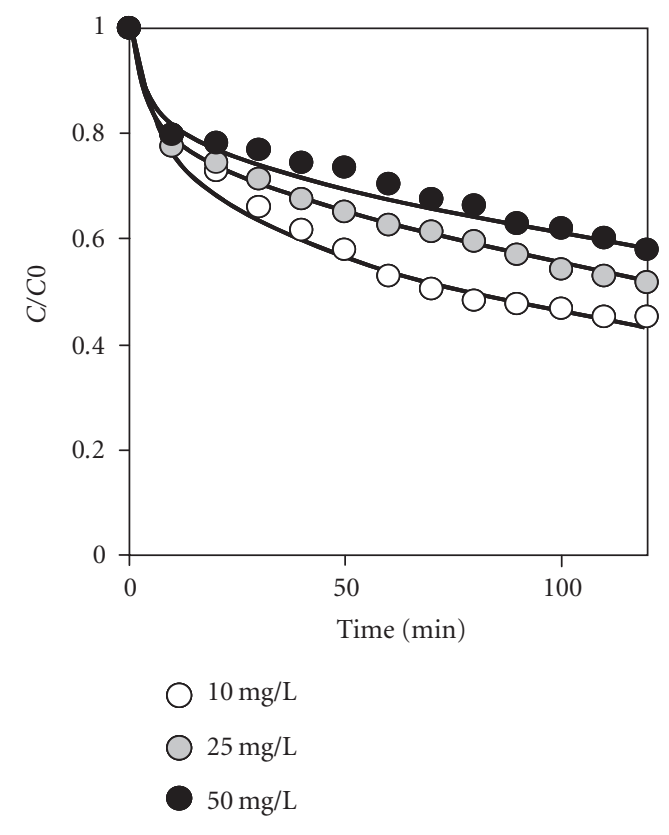

FIGURE 16: Solar photocatalytic decolorization of Drimarene Yellow, effect of the concentration, $\mathrm{TiO}_{2} 2.8 \mathrm{mg} \cdot \mathrm{cm}^{-2}$.

irradiation time. This suggests the development of the technique in sun-bathed countries, especially in developing countries. The effect of concentration has to be clarified as it differs much in the case of Orange II or Drimarene dyes. Decolorization does not imply the total destruction of the molecule and some intermediates are likely present when the original dye has vanished. In a recent study [16], it has been demonstrated that photocatalytic treatment of an industrial wastewater containing Drimarene dyes enhances the biodegradability of waste since it leads to a higher ratio of BOD to COD showing a synergy between photocatalytic and biological treatments.

\section{ACKNOWLEDGMENTS}

Authors are grateful to Mrs. Christine Monnet, Project Manager at Ahlstrom Research Corporate Centre, for the generous donation of the photocatalyst. The sponsorship of the French-Moroccan Committee is also acknowledged (MA/02/49).

\section{REFERENCES}

[1] J. Wu, M. A. Eiteman, and S. E. Law, "Evaluation of membrane filtration and ozonation processes for treatment of reactive-dye wastewater," Journal of Environmental Engineering, vol. 124, no. 3, pp. 272-277, 1998.

[2] O. Legrini, E. Oliveros, and A. M. Braun, "Photochemical processes for water treatment," Chemical Reviews, vol. 93, no. 2, pp. 671-698, 1993.

[3] C. Galindo, P. Jacques, and A. Kalt, "Photodegradation of the aminoazobenzene acid orange 52 by three advanced oxidation processes: $\mathrm{UV} / \mathrm{H}_{2} \mathrm{O}_{2}, \mathrm{UV} / \mathrm{TiO}_{2}$ and $\mathrm{VIS} / \mathrm{TiO}_{2}$. Comparative mechanistic and kinetic investigations," Journal of Photochemistry and Photobiology A: Chemistry, vol. 130, no. 1, pp. 35-47, 2000.

[4] O. d'Hennezel, P. Pichat, and D. F. Ollis, "Benzene and toluene gas-phase photocatalytic degradation over $\mathrm{H}_{2} \mathrm{O}$ and HCL pretreated $\mathrm{TiO}_{2}$ : by-products and mechanisms," Journal of Photochemistry and Photobiology A: Chemistry, vol. 118, no. 3, pp. 197-204, 1998.

[5] H. Y. Chen, O. Zahraa, M. Bouchy, F. Thomas, and J. Y. Bottero, "Adsorption properties of $\mathrm{TiO}_{2}$ related to the photocatalytic degradation of organic contaminants in water," Journal of Photochemistry and Photobiology A: Chemistry, vol. 85, no. 1-2, pp. 179-186, 1995.

[6] A. Mills, C. E. Holland, R. H. Davies, and D. Worsley, "Photomineralization of salicylic acid: a kinetic study," Journal of Photochemistry and Photobiology A: Chemistry, vol. 83, no. 3, pp. 257-263, 1994.

[7] S. Tunesi and M. Anderson, "Influence of chemisorption on the photodecomposition of salicylic acid and related compounds using suspended titania ceramic membranes," Journal of Physical Chemistry, vol. 95, no. 8, pp. 3399-3405, 1991.

[8] C. Kormann, D. W. Bahnemann, and M. R. Hoffmann, "Photolysis of chloroform and other organic molecules in aqueous $\mathrm{TiO}_{2}$ suspensions," Environmental Science and Technology, vol. 25, no. 3, pp. 494-500, 1991.

[9] Degussa Corp. Technical Bulletin Pigments, No. 56, 5th Ed., Degussa AG, Frankfurt, G. 1990.

[10] H. Y. Chen, O. Zahraa, and M. Bouchy, "Inhibition of the adsorption and photocatalytic degradation of an organic contaminant in an aqueous suspension of $\mathrm{TiO}_{2}$ by inorganic ions," Journal of Photochemistry and Photobiology A: Chemistry, vol. 108, no. 1, pp. 37-44, 1997.

[11] C. Hachem, F. Bocquillon, O. Zahraa, and M. Bouchy, "Decolourization of textile industry wastewater by the photocatalytic degradation process," Dyes and Pigments, vol. 49, no. 2, pp. 117-125, 2001. 
[12] S. M. Ould-Mame, O. Zahraa, and M. Bouchy, "Photocatalytic degradation of salicylic acid on fixed $\mathrm{TiO}_{2}$ - kinetic studies," International Journal of Photoenergy, vol. 2, no. 2, pp. 59-66, 2000.

[13] O. Zahraa, C. Dorion, S. M. Ould-Mame, and M. Bouchy, "Titanium dioxide deposit films for photocatalytic studies of water pollutants," Journal of Advanced Oxidation Technologies, vol. 4, no. 1, pp. 40-46, 1999.

[14] W. A. Jacoby, D. M. Blake, J. A. Fennell, et al., "Heterogeneous photocatalysis for control of volatile organic compounds in indoor air," Journal of the Air and Waste Management Association, vol. 46, no. 9, pp. 891-898, 1996.

[15] P. Wyness, J. F. Klausner, D. Y. Goswami, and K. S. Schanze, "Performance of nonconcentrating solar photocatalytic oxidation reactors part 1: flat-plate configuration," Journal of Solar Energy Engineering, vol. 116, no. 1, pp. 2-7, 1994.

[16] A. Alinsafi, "Traitabilité de rejets liquides de l'industrie textile," Ph.D. thesis in Processes Engineering, INPL, Nancy, France, 2004. 


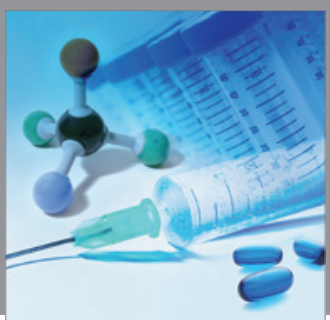

International Journal of

Medicinal Chemistry

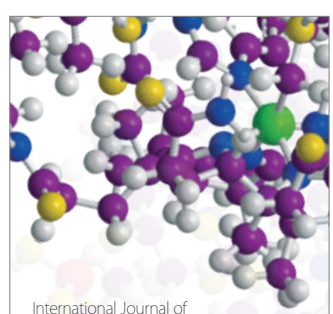

Carbohydrate Chemistry

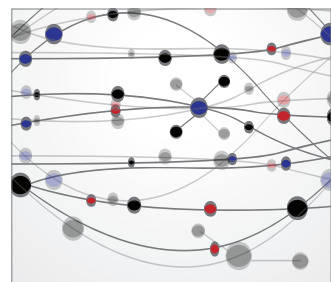

The Scientific World Journal
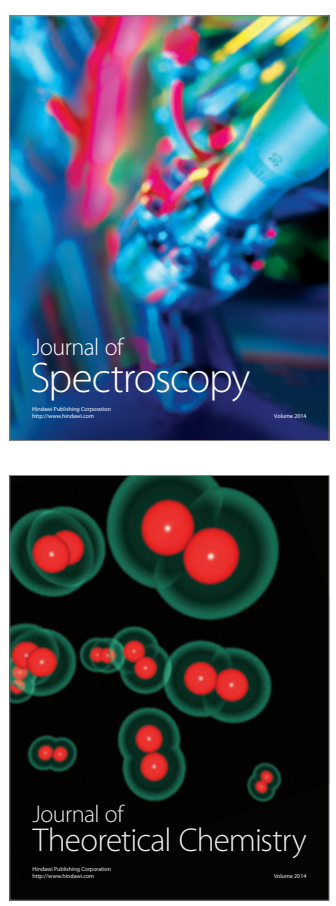
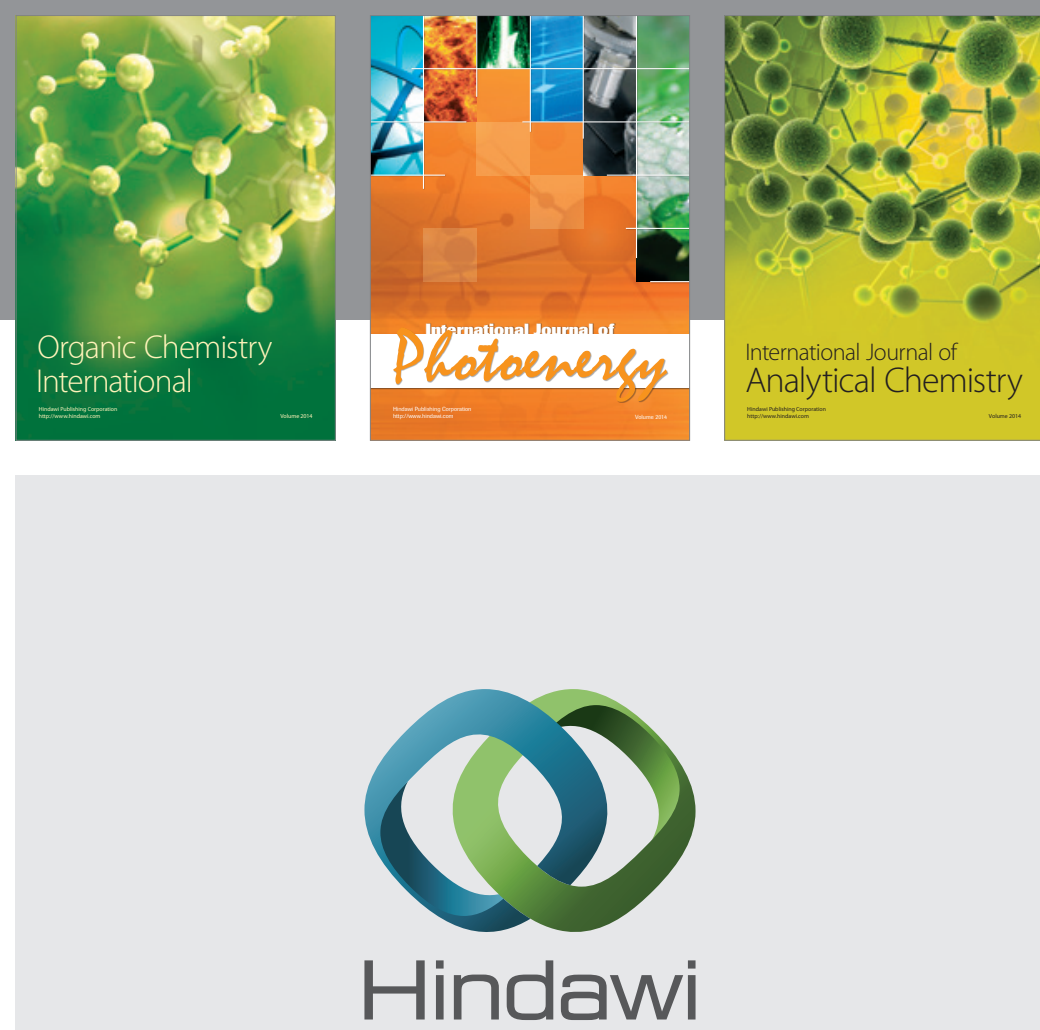

Submit your manuscripts at

http://www.hindawi.com
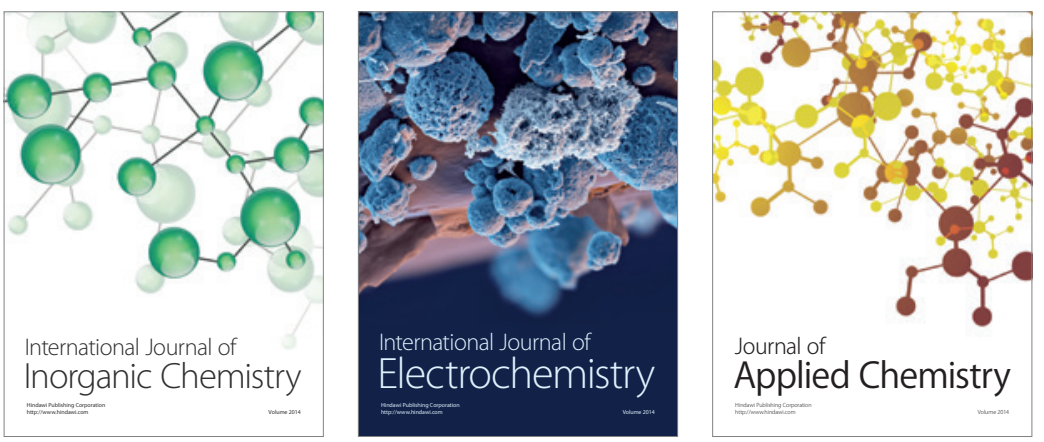

Journal of

Applied Chemistry
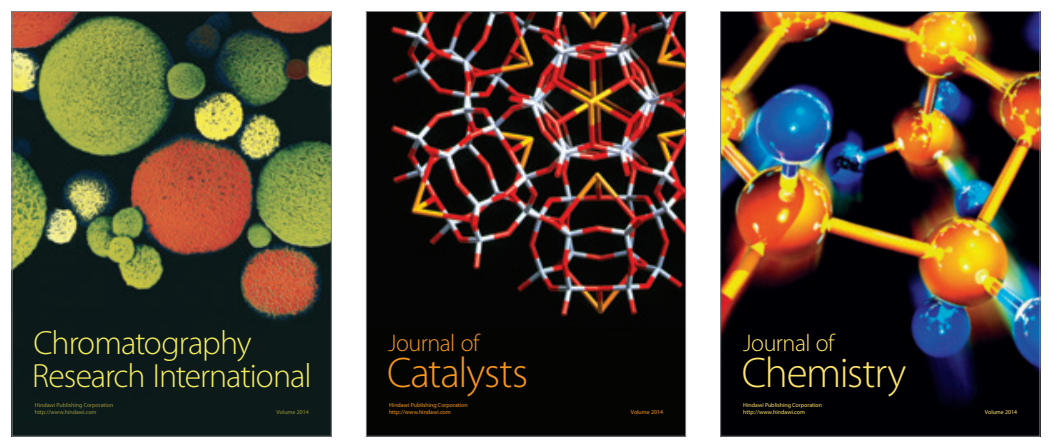
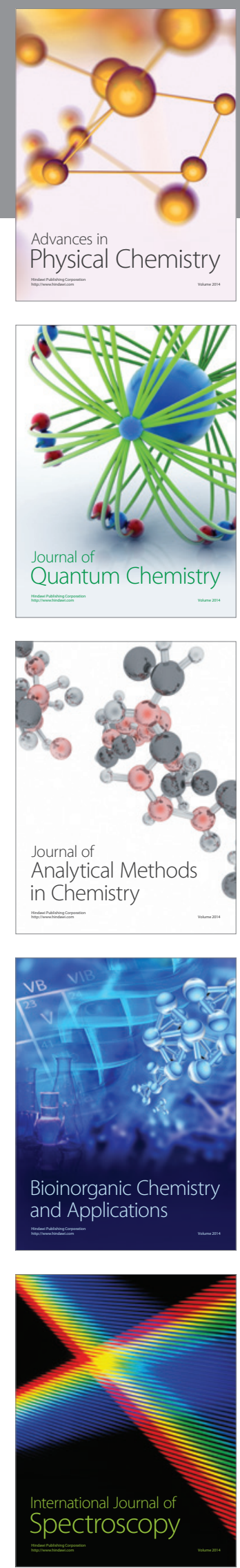